\title{
Extended Target Shape Estimation by Fitting B-Spline Curve
}

\author{
Jin-long Yang, Peng Li, and Hong-wei Ge \\ School of Internet of Things Engineering, Jiangnan University, Wuxi 214122, China \\ Correspondence should be addressed to Jin-long Yang; yjlgedeng@163.com
}

Received 8 February 2014; Revised 10 June 2014; Accepted 11 June 2014; Published 25 June 2014

Academic Editor: Zhihua Zhang

Copyright (C) 2014 Jin-long Yang et al. This is an open access article distributed under the Creative Commons Attribution License, which permits unrestricted use, distribution, and reproduction in any medium, provided the original work is properly cited.

\begin{abstract}
Taking into account the difficulty of shape estimation for the extended targets, a novel algorithm is proposed by fitting the $\mathrm{B}$-spline curve. For the single extended target tracking, the multiple frame statistic technique is introduced to construct the pseudomeasurement sets and the control points are selected to form the B-spline curve. Then the shapes of the extended targets are extracted under the Bayes framework. Furthermore, the proposed shape estimation algorithm is modified suitably and combined with the probability hypothesis density (PHD) filter for multiple extended target tracking. Simulations show that the proposed algorithm has a good performance for shape estimate of any extended targets.
\end{abstract}

\section{Introduction}

In the traditional low resolution sensor system, each target is tracked as a single point source; that is, its extension is assumed to be neglectable in comparison with sensor resolution. With the increase of the resolution of modern radars and other detection equipment, the echo signal of a target may be distributed in a different range resolution cell; thus, the measurement is no longer equivalent to a point; that is, a single target may generate multiple measurements. Such target is referred to as an extended target in [1-4]. Recently, extended target tracking (ETT) is a hot topic in the field including the short-range applications or maritime surveillance, which has drawn a considerable attention [5-9].

In the conventional extended target tracking, the measurements are modeled as a spatial distribution model in [1], and two examples-a point target with more measurement sources and an object with infinitely thin stick-are used to prove the effectiveness of the approach. Poisson process with a spatially dependent rate parameter is introduced in [2], assuming that each target produces measurements with Poisson distributed random number. It is considered that in this measurement model, the target is sufficiently far away from the sensor, and the measurements resemble a point cluster rather than a geometric structure [7]. Random matrix (RM) is proposed in [8], which has been used to track elliptical target extension [9]. Another method is random hypersurface model (RHM) [3] which is employed for modeling the target extent. However, these methods can only effectively achieve the shape estimate for the target with similar ellipse shape. They cannot effectively estimate the irregular shape of the extended target. In [4], star-convex target extension estimation method is proposed based on RHM under the condition that a measurement source is assumed to be an element of a randomly scaled version of the shape boundary. Moreover, the one-dimension probability density needs to be specified in advance in star-convex shape estimation and it is assumed to be independent of the shape.

To solve the aforementioned problem, a novel shape estimation algorithm based on the B-spline curve fitting is proposed in this paper, and then the proposed shape estimation method is integrated into the framework of extended target probability hypothesis density (ET-PHD) filter for multiple extended target tracking $[5,6]$. Simulations show that the proposed algorithm has a good performance for shape estimate of any extended targets.

\section{Backgrounds}

2.1. Kalman Filter. Assume the state equation and the measurement equation of a single target in two-dimensional plane are given by

$$
\begin{gathered}
x_{k+1}=F x_{k}+G w_{k} \\
y_{k}=H x_{k}+v_{k},
\end{gathered}
$$


where $x_{k}$ and $y_{k}$ are the state vector and the observation vector at time $k$, respectively. $F$ and $H$ are the transition matrix and the measurement matrix, respectively. $w_{k}$ and $v_{k}$ are the process noise and the observation noise and are uncorrelated Gaussian white noise vectors with covariance matrixes $Q_{k}$ and $R_{k}$, respectively.

Suppose that $x_{k \mid k}$ and the covariance $P_{k \mid k}$ are optimal estimations at time $k$ in the fusion center; then, the recursive steps of the Kalman Filter (KF) at time $k+1$ are as follows [10].

(1) Prediction of state and covariance:

$$
\begin{gathered}
x_{k+1 \mid k}=F x_{k \mid k}, \\
P_{k+1 \mid k}=F P_{k \mid k} F^{T}+G Q_{k} G^{T} .
\end{gathered}
$$

(2) Calculating gain:

$$
\begin{aligned}
& K_{k+1}=P_{k+1 \mid k} H^{T}\left[S_{k+1 \mid k}\right]^{-1}, \\
& S_{k+1 \mid k}=H P_{k+1 \mid k} H^{T}+R_{k+1} .
\end{aligned}
$$

(3) Update of state and covariance:

$$
\begin{aligned}
& x_{k+1 \mid k+1}=x_{k+1 \mid k}+K_{k+1}\left(y_{k+1}-H x_{k+1 \mid k}\right), \\
& P_{k+1 \mid k+1}=\left[I-K_{k+1} H\right] P_{k+1 \mid k} .
\end{aligned}
$$

2.2. The B-Spline Curve. Assume that readers are familiar with the concepts of B-spline curves. A smooth subsection curve can be obtained by fitting the control point set. The Bspline curve of order $l$ can be described as [11]

$$
S(t)=\sum_{i=1}^{n} \mu_{i} \cdot N_{i, l}(t),
$$

where $\mu_{i}$ in $U_{k}=\left[\mu_{1}, \mu_{2}, \ldots, \mu_{n}\right]^{T}$ is a control point and $N_{i, l}(t)$ is the B-spline basis function, which is defined over a knot vector $T=\left\{t_{0}, \ldots, t_{n+l}\right\}$. The basis function can be recursively defined as $[11,12]$

$$
\begin{aligned}
& N_{i, l}(t)=\frac{t-t_{i}}{t_{i+l-1}-t_{i}} N_{i, l-1}(t)+\frac{t_{i+l}-t}{t_{i+l}-t_{i+1}} N_{i+1, l-1}(t), \\
& N_{i, 1}(t)= \begin{cases}1, & \text { if } t_{i} \leq t \leq t_{i+1} \\
0, & \text { otherwise. }\end{cases}
\end{aligned}
$$

When $l=3$, we can obtain that

$$
N_{i, 3}(t)= \begin{cases}\frac{\left(t-t_{i}\right)^{2}}{\left(t_{i+1}-t_{i}\right)\left(t_{i+2}-t_{i}\right)}, & t \in\left[t_{i}, t_{i+1}\right) \\ \frac{\left(t-t_{i}\right)\left(t_{i+2}-t\right)}{\left(t_{i+2}-t_{i}\right)\left(t_{i+2}-t_{i+1}\right)} & \\ +\frac{\left(t-t_{i+1}\right)\left(t_{i+3}-t\right)}{\left(t_{i+2}-t_{i+1}\right)\left(t_{i+3}-t_{i+1}\right)}, & t \in\left[t_{i+1}, t_{i+2}\right) \\ \frac{\left(t_{i+3}-t\right)^{2}}{\left(t_{i+3}-t_{i+1}\right)\left(t_{i+3}-t_{i+2}\right)}, & t \in\left[t_{i+2}, t_{i+3}\right) \\ 0, & \text { otherwise. }\end{cases}
$$

\section{Single Extended Target Shape Estimation}

In this section, the Bayesian filter framework is introduced for single extended target state and shape estimates. Assume the state equation and the measurement equation are the same as (1) and (2). We define $x_{k}$ as the center of the extended target and $X_{k}$ as the control matrix including the shape information. They are not related to each other. Thus we can estimate them with two parallel KFs, which can modify them by recursion update. And the detailed steps of the proposed algorithm are described as follows.

Step 1. At time $k=0$, initialize parameters $x_{0 \mid 0}, P_{0 \mid 0}, X_{0 \mid 0}$, and $\Delta_{0 \mid 0}$, where $P_{0 \mid 0}$ and $\Delta_{0 \mid 0}$ denote the state covariance and the shape covariance, respectively.

Step 2. When $k \geq 1$, implement the KF for state estimate:

(2.1) prediction of the state and covariance according to (3) and (4), respectively;

(2.2) update of the state by the latest measurement set $Y_{k+1}$ :

$$
x_{k+1 \mid k+k}=x_{k+1 \mid k}+K_{k+1}\left(\frac{1}{\left|Y_{k+1}\right|} \sum_{i=1}^{\left|Y_{k+1}\right|} y_{k+1, i}-H x_{k+1 \mid k}\right) \text {, }
$$

where $Y_{k+1}=\left\{y_{k+1, i}\right\}_{i=1, \ldots, n}$ and $\left|Y_{k+1}\right|$ denotes the number of the measurements at time $k+1 . y_{k+1, i}$ denotes the $i$ th measurement in $Y_{k+1}$. The covariance $P_{k+1 \mid k+1}$ can be updated by (7).

Step 3. Construct a new pseudomeasurement set $Z_{k+1}$.

(3.1) Let $\widetilde{Y}_{k+1}=\left\{y_{k+1, i}-H x_{k+1 \mid k} \mid y_{k+1, i} \in Y_{k+1}\right\}_{i=1}^{\left|Y_{k+1}\right|}$, and then add $\tilde{Y}_{k+1}$ to the pseudomeasurement set $Z_{k}$, that is, $\widehat{Z}_{k+1}=Z_{k} \cup \tilde{Y}_{k+1}$.

(3.2) Set $m$ as the largest number of pseudomeasurements. If $\left|\widehat{Z}_{k+1}\right| \leq m$, then let the new pseudomeasurement set $Z_{k+1}=\widehat{Z}_{k+1}$. Otherwise, delete the $\left|\widehat{Z}_{k+1}\right|-m$ foregoing measurements from $\widehat{Z}_{k+1}$, and then let $Z_{k+1}=\widehat{Z}_{k+1}$.

Step 4. Update the shape of the extended target by the pseudomeasurement set $Z_{k+1}$.

(4.1) Divide the interval $[0,2 \pi]$ into $n$ equal angles, and generate a fixed angle set $\aleph=\left\{\theta_{i}\right\}_{i=1}^{n}$. Then partition the pseudomeasurement set by the angles, and define $D_{k+1, i}$ as the measurement set in the area of the $i$ th partition. That is,

$$
\begin{aligned}
D_{k+1, i} & =\left\{z_{k+1, j} \mid d_{L_{i}}\left(z_{k+1, j}\right)<d, C\left(z_{k+1, j}\right)=\text { ture }\right\}, \\
d_{L_{i}}\left(z_{k+1, j}\right) & =\frac{\left\|B_{1} z_{k+1, j}^{(1)}+B_{2} z_{k+1, j}^{(2)}\right\|}{\sqrt{B_{1}^{2}+B_{2}^{2}}},
\end{aligned}
$$




$$
C\left(z_{k+1, j}\right)=\left\{\begin{array}{rr}
\text { true, } \quad & \bar{L}_{i}: A_{1} z_{k+1, j}^{(1)}+A_{2} z_{k+1, j}^{(2)}>0 \\
& \theta_{i} \in(0, \pi] \\
& \text { or } \bar{L}_{i}: A_{1} z_{k+1, j}^{(1)}+A_{2} z_{k+1, j}^{(2)}<0 \\
& \theta_{i} \in[\pi, 2 \pi)
\end{array}\right.
$$

where $d$ is the width of partition. $z_{k+1, j} \in Z_{k+1}, z_{k+1, j}=$ $\left[z_{k+1, j}^{(1)}, z_{k+1, j}^{(2)}\right], z_{k+1, j}^{(1)}$, and $z_{k+1, j}^{(2)}$ denote the position of $x$ - and $y$-coordinate at time $k+1 . L_{i}$ denotes a line through the origin of coordinate and along the $i$ th partition angle direction. $d_{L_{i}}\left(z_{k+1, j}\right)$ denotes the perpendicular distance between $z_{k+1, j}$ and line $L_{i}$. The parameters $B_{1}$ and $B_{2}$ of line $L_{i}$ satisfy $B_{1} / B_{2}=-\tan \left(\theta_{i}\right) . \bar{L}_{i}$ denotes a line through the origin of coordinate, and it is perpendicular to line $L_{i}$. The parameters $A_{1}$ and $A_{2}$ of line $\bar{L}_{i}$ satisfy $A_{2} / A_{1}=\tan \left(\theta_{i}\right)$. Figure 1 shows the $i$ th angle partition and the measurements in the area enclosed by points $\mathrm{O} 1, \mathrm{O} 2, \mathrm{O} 3$, and $\mathrm{O} 4$ which constitute the measurement set $D_{k+1, i}$.

(4.2) Calculate $E\left(\left\|D_{k, i}\right\|_{L_{i}}\right)$ which is the expectation of the perpendicular distance between the elements of $D_{k+1, i}$ and line $\bar{L}_{i}$. Then the radial extension distance $\rho_{k+1, i}$ can be obtained by

$$
\rho_{k+1, i}=2 \frac{1}{\left|D_{k+1, i}\right|} \sum_{j=1}^{\left|D_{k+1, i}\right|} \frac{\left\|A_{1} z_{k+1, j}^{(1)}+A_{2} z_{k+1, j}^{(2)}\right\|}{\sqrt{A_{1}^{2}+A_{2}^{2}}} .
$$

In this paper, we set the $\rho_{k+1, i}$ as the control point of the extended target shape parameter and define $X_{k \mid k}=$ $\left[\left\{\rho_{k, i}\right\}_{i=1}^{n}\right]^{T}$ as the control matrix of the shape, which includes the shape information.

(4.3) Shape estimation by implementing the onedimension (1-D) KF: assume the shape control matrix $X_{k \mid k}$ and the shape covariance $\Delta_{k \mid k}$ have been obtained. Then the recursion steps of shape estimates are as follows.

Prediction:

$$
\begin{aligned}
& X_{k+1 \mid k}=X_{k \mid k}, \\
& \Delta_{k+1 \mid k}=\Delta_{k \mid k}+\bar{H}\left\|R_{k+1}\right\|,
\end{aligned}
$$

where $\bar{H}=\underbrace{[1,1, \ldots, 1]^{T}}_{n}$ and $R_{k+1}$ denotes the measurement noise covariance.

Update:

$$
\begin{gathered}
X_{k+1 \mid k+1}=X_{k+1 \mid k}+\left(X_{k \mid k}-\widehat{K}_{k+1} \cdot 2 E\left(\left\|D_{k+1, i}\right\|\right)\right), \\
\Delta_{k+1 \mid k+1}=\left(I-\widehat{K}_{k+1} \bar{H}^{T}\right) \Delta_{k+1 \mid k} \\
\widehat{K}_{k+1}=\operatorname{diag}\left(\left\{\frac{\delta_{k+1 \mid k, i}}{\delta_{k+1 \mid k, i}+v_{k+1}}\right\}_{i=1}^{n}\right),
\end{gathered}
$$

where the shape covariance $\Delta_{k+1 \mid k+1}=\left[\delta_{k+1, i}\right]_{n \times 1} \cdot v_{k+1}$ denotes the measurement noise.

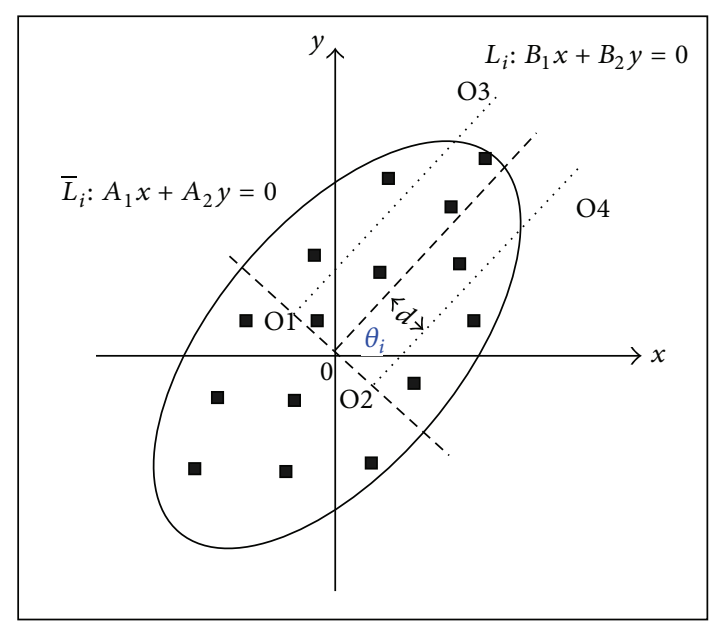

- Measurement

Figure 1: Angle interval partition.

Step 5. Shape estimation according to $X_{k+1 \mid k+1}$ and the Bspline curve fitting technique.

(5.1) Map the control points to the Cartesian coordinates by

$$
\begin{gathered}
U_{k+1}=\left\{\mu_{i}\right\}_{i=1}^{n}=\widehat{\aleph} X_{k+1 \mid k+1}, \\
\widehat{\aleph}=\left[\begin{array}{c}
\cos \left(\theta_{i}\right), \ldots, \cos \left(\theta_{n}\right) \\
\sin \left(\theta_{i}\right), \ldots, \sin \left(\theta_{n}\right)
\end{array}\right]_{2 \times n} .
\end{gathered}
$$

(5.2) Produce a closed control point set by adding the element $\mu_{1}, \mu_{2}, \mu_{3}$ to the end of the $U_{k+1}$, and describe it as $\underline{U}_{k+1}=\left\{\underline{\mu}_{i}\right\}_{i=1}^{n+3}$. Then the closed cubic B-spline curve can be obtained by

$$
S(t)=\sum_{i=1}^{n+3} \underline{\mu}_{k+1} \cdot N_{i, 3}(t) .
$$

\section{Multiple Extended Target Shape Estimation}

4.1. Multiple Extended Target PHD Filter. The standard PHD filter for single measurement target tracking has been described in [13-15]; however, it is not suitable for METT. Recently, Mahler has derived a correct equation for extended target PHD (ET-PHD) filter based on the Poisson multitarget measurement model [5]. The Gaussian mixture implement of the multiple extended target PHD filter is presented in [6] and referred to as ET-GM-PHD filter. The detailed filter process of ET-GM-PHD can refer to $[6,16,17]$.

4.2. Multiple Extended Target Tracking Algorithm. In this section, we combine the proposed shape estimation algorithm into the framework of ET-GM-PHD filter, which can effectively achieve the multiple extended target tracking with different shape estimation. We refer to this algorithm as Shape-ET-GM-PHD, and its steps are as follows. 
(1) Prediction. Assume that the state vector $x$ and its shape vector $X$ are independent, and the survival and detection probabilities are independent of them; that is, $p_{S, k}(x, X)=$ $p_{S}$ and $p_{D, k}(x, X)=p_{D}$. Assume that $v_{k-1}(x, X)$ denotes the joint posterior PHD function at time $k-1$ and is approximated by the Gaussian mixture distributions. Then the predicted PHD function $v_{k \mid k-1}(x, X)$ can be described as

$$
\begin{aligned}
& v_{k \mid k-1}(x, X) \\
& =p_{S} \iint p_{k \mid k-1}\left(x, X \mid x^{\prime}, X^{\prime}\right) v_{k-1 \mid k-1}(x, X) d x^{\prime} d X^{\prime} \\
& =p_{S} \iint p_{k \mid k-1}\left(x \mid x^{\prime}, X^{\prime}\right) p_{k \mid k-1}\left(X \mid X^{\prime}\right) \\
& \quad \times v_{k-1 \mid k-1}(x, X) d x^{\prime} d X^{\prime} \\
& \quad+p_{S} \sum_{j=1}^{J_{k \mid k-1}} w_{k-1 \mid k-1} \int N\left(x_{k-1 \mid k-1} \mid m_{k-1 \mid k-1}^{(j)}, P_{k-1 \mid k-1}^{(j)}\right. \\
& \quad \int \Psi\left(X_{k-1 \mid k-1} \mid \widetilde{X}_{k-1 \mid k-1}, \Delta_{k-1 \mid k-1}\right) p_{k \mid k-1}\left(X \mid X^{\prime}\right) d X^{\prime},
\end{aligned}
$$

where

$$
\begin{aligned}
& \int N\left(x_{k-1 \mid k-1} \mid m_{k-1 \mid k-1}^{(j)}, P_{k-1 \mid k-1}^{(j)}+\bar{\sigma}_{k-1 \mid k-1} \widetilde{I}\right) \\
& \quad \times p_{k \mid k-1}\left(x \mid x^{\prime}, X^{\prime}\right) d x^{\prime} \\
& =N\left(x_{k \mid k-1} \mid m_{k \mid k-1}^{(j)}, P_{k \mid k-1}^{(j)}+\bar{\sigma}_{k \mid k-1} \widetilde{I}\right), \\
& \int \Psi\left(X_{k-1 \mid k-1} \mid \widetilde{X}_{k-1 \mid k-1}, \Delta_{k-1 \mid k-1}\right) p_{k \mid k-1}\left(X \mid X^{\prime}\right) d X^{\prime} \\
& =\prod_{i=1}^{N u m} N\left(X_{k \mid k-1}^{(i)} \mid \widetilde{X}_{k \mid k-1}^{(j, i)}, \Delta_{k \mid k-1}^{(j, i)}\right)
\end{aligned}
$$

$\widetilde{I}=\operatorname{diag}[1,1,0,0]$, and $\bar{\sigma}_{k-1 \mid k-1}$ denotes the mean of the shape standard variance, One has $m_{k \mid k-1}^{(j)}=F_{k \mid k-1} m_{k-1 \mid k-1}^{(j)}, P_{k \mid k-1}^{(j)}=$ $F_{k \mid k-1} P_{k-1 \mid k-1}^{(j)} F_{k \mid k-1}^{T}+Q_{k \mid k-1}$. Num denotes the divided number of the shape area according to the degree of angle. $\widetilde{X}_{k \mid k-1}^{(j, i)}=$ $\widetilde{X}_{k-1 \mid k-1}^{(j, i)}$ and $\Delta_{k \mid k-1}^{(j, i)}=\Delta_{k-1 \mid k-1}^{(j, i)}+\mathbb{Q}_{k}$ denote the control matrix of shape and its predicted covariance, and $\mathbb{Q}_{k}$ denotes the shape process noise covariance. $\widetilde{X}_{k \mid k-1}^{(j, i)}$ and $\Delta_{k \mid k-1}^{(j, i)}$ can also be described as $\widetilde{X}_{k \mid k-1}^{(j, i)}=\left[\left\{\widetilde{\rho}_{k \mid k-1}^{(j, i)}\right\}_{i=1}^{\text {Num }}\right]^{T}$ and $\Delta_{k \mid k-1}^{(j, i)}=$ $\left[\left\{\sigma_{k \mid k-1}^{(j, i)}\right\}_{i=1}^{\text {Num }}\right]^{T}$, where the definition of $\widetilde{\rho}_{k \mid k-1}^{(j, i)}$ is the same as that of (13). $\sigma_{k \mid k-1}^{(j, i)}$ denotes the predicted variance of the shape.

(2) Update. The updated formula of the intensity function can be described as

$$
v_{k \mid k}(x, X)=L_{Z_{k}}(x, X) v_{k \mid k-1}(x, X),
$$

where $v_{k \mid k}(x, X)$ denotes an intensity function of the state $x$ with shape information $X$ and $L_{Z_{k}}(x, X)$ denotes the pseudolikelihood function. $Z_{k}$ denotes the measurement set at time $k$. When $Z_{k}=\emptyset$,

$$
L_{Z_{k}}(x, X) \triangleq 1-p_{D}(x, X)+e^{-r(x, X)} p_{D}(x, X) ;
$$

otherwise,

$$
\begin{aligned}
L_{Z_{k}}(x, X) \triangleq & 1-\left(1-e^{-r(x, X)}\right) p_{D}(x, X)+e^{-r(x, X)} p_{D}(x, X) \\
& \times \sum_{P \angle Z_{k}} \omega_{P} \sum_{W \in P} \frac{r(x, X)^{|W|}}{d_{W}} \prod_{z \in W} \frac{\phi_{z}(x, X)}{\lambda_{k} c_{k}},
\end{aligned}
$$

where $P \angle Z_{k}$ denotes a partition subset $P$ of the measurement set $Z_{k}, W$ denotes a subset of a partition $P$, and $\cup_{W \in P} W=$ $Z_{k} \cdot \phi_{z}(x, X)$ denotes the measurement likelihood function of one measurement originating from an extended target $x$, $r(x, X)$ is the measurement expectation, and $p_{D}(x, X)=p_{D}$ denotes the detection probability of the sensor. Clutter has a Poisson distribution, and its density can be described as $\beta_{F A, k}=\lambda_{k} c_{k}\left(z_{k}\right), \lambda_{k}$ denotes the mean number of clutter measurements, and $c_{k}\left(z_{k}\right)$ is the space distribution of the clutter. Consider

$$
\begin{aligned}
& \omega_{P}=\frac{\prod_{W \in P} d_{W}}{\sum_{P^{\prime} \angle Z_{k}} \prod_{W \in P^{\prime}} d_{W^{\prime}}}, \\
& d_{W}=\delta_{|W|, 1}+v_{k \mid k-1}\left[e^{-r} r^{|W|} p_{d} \prod_{z \in W} \frac{\phi_{z}}{\beta_{F A, k}}\right],
\end{aligned}
$$

where $\delta_{i, j}$ denotes the Kronecker delta function and $r=$ $r(x, X) . \beta_{F A, k}$ denotes the clutter distribution of the measurement space.

In the following subsections, assume that the current estimated PHD $v_{k \mid k}(x, X)$ can be approximated as a Gaussian mixture distribution. The corrected PHD can be described as

$$
v_{k \mid k}(x, X)=v_{k \mid k}^{N D}(x, X)+\sum_{p \in Z} \sum_{W \in p} v_{k \mid k}^{D}(x, X, W)
$$

where $v_{k \mid k}^{N D}(x, X)$ denotes the PHD of the targets without detecting cases, and it can be described as

$$
\begin{aligned}
v_{k \mid k}^{N D}(x, X)= & \sum_{j=1}^{J_{k \mid k-1}} w_{N D, k \mid k}^{(j)} N\left(x_{k \mid k} \mid m_{k \mid k}^{(j)}, P_{k \mid k}^{(j)}+\bar{\sigma}_{k \mid k}^{(j)} \widetilde{I}\right) \\
& \times \prod_{i=1}^{N u m} N\left(X_{k \mid k}^{(i)} \mid \widetilde{X}_{k \mid k}^{(j, i)}, \Delta_{k \mid k}^{(j, i)}\right),
\end{aligned}
$$

where $m_{k \mid k}^{(j)}=m_{k \mid k-1}^{(j)}, P_{k \mid k}^{(j)}=P_{k \mid k-1}^{(j)}, \Delta_{k \mid k}^{(j, i)}=\Delta_{k \mid k-1}^{(j, i)}$, and $\widetilde{X}_{k \mid k}^{(j, i)}=\widetilde{X}_{k \mid k-1}^{(j, i)}$. 
$v_{k \mid k}^{D}(x, X, W)$ denotes the PHD of the detected target cases and can be described as

$$
\begin{aligned}
v_{k \mid k}^{D}(x, X, W)= & \sum_{j=1}^{J_{k \mid k-1}} w_{k \mid k}^{(j)} N\left(x_{k} \mid m_{k \mid k}^{(j)}, P_{k \mid k}^{(j)}+\bar{\sigma}_{k \mid k}^{(j)} \widetilde{I}\right) \\
& \times \prod_{i=1}^{\text {Num }} N\left(X_{k}^{(i)} \mid \widetilde{X}_{k \mid k}^{(j, i)}, \Delta_{k \mid k}^{(j, i)}\right),
\end{aligned}
$$

where

$$
\begin{aligned}
& m_{k \mid k}^{(j)}=m_{k \mid k-1}^{(j)}+K_{k}\left(\bar{z}_{k}^{W}-H m_{k \mid k-1}^{(j)}\right), \\
& \bar{z}_{k}^{W}=\frac{1}{|W|} \sum_{z_{k}^{(l)} \in W} z_{k}^{(l)} \\
& P_{k \mid k}^{(j)}=\left(I-K_{k} H\right) P_{k \mid k-1}^{(j)}, \\
& S_{k}=P_{k \mid k-1}^{(j)}+\bar{\sigma}_{k \mid k}^{(j)}\left[\begin{array}{ll}
1 & 0 \\
0 & 0
\end{array}\right] \otimes I_{2}, \\
& K_{k}=P_{k \mid k-1}^{(j)} H^{T}\left(S_{k}\right)^{-1} \text {, } \\
& \bar{\sigma}_{k \mid k}^{(j)}=\frac{1}{\operatorname{Num}} \sum_{i=1}^{\mathrm{Num}} \sigma_{k \mid k}^{(j, i)}, \\
& w_{k}^{(j)}=\frac{w_{p}}{d_{W}} e^{-\gamma^{(j)}}\left(\frac{\gamma^{(j)}}{\beta_{F A, k}}\right)^{|W|} p_{D} L_{k}^{(j, W)} w_{k \mid k-1}^{(j)}, \\
& w_{p}=\frac{\prod_{W \in p} d_{W}}{\sum_{p^{\prime} \angle Z^{\prime}} \prod_{W^{\prime} \in p^{\prime}} d_{W^{\prime}}}, \\
& d_{W}=\delta_{|W|, 1}+\sum_{\ell=1}^{J_{k \mid k-1}} e^{-\gamma^{(\ell)}}\left(\frac{\gamma^{(j)}}{\beta_{F A, k}}\right)^{|W|} p_{D} L_{k}^{(\ell, W)} w_{k \mid k-1}^{(\ell)} \text {, } \\
& L_{k}^{(j, W)}=\prod_{i=1}^{\mathrm{Num}} \widehat{L}_{k}^{(j, W, i)} \\
& \widehat{L}_{k}^{(j, W, i)}= \begin{cases}\left(\widetilde{L}_{k}^{(j, W, i)}\right)^{\left|W^{(i)}\right| / \mathrm{Num},}, & \left|W^{(i)}\right| \neq 0 \\
\alpha \cdot\left(\widetilde{L}_{k}^{(j, W, i)}\right)^{1 / \mathrm{Num}}, & \left|W^{(i)}\right|=0,\end{cases} \\
& \widetilde{L}_{k}^{(j, W, i)}=\frac{1}{\sqrt{2 \pi} \sigma_{k \mid k-1}^{(j, i)}} \cdot \exp \left(-\frac{1}{2 \sigma_{k \mid k-1}^{2(j, i)}}\left(\tilde{\rho}_{k+1 \mid k}^{(j, i)}-\widetilde{\rho}^{(W, i)}\right)^{2}\right),
\end{aligned}
$$

where $\alpha$ denotes the penalty coefficient. The shape parameters can be obtained by

$$
\begin{aligned}
\Delta_{k \mid k}^{(j, i)} & =\left(I_{\mathrm{Num}}-\widetilde{K}_{k} \bar{H}^{T}\right) \Delta_{k \mid k-1}^{(j, i)}, \\
\widetilde{X}_{k \mid k}^{(j, i)} & =\widetilde{X}_{k \mid k-1}^{(j, i)}+\widetilde{K}_{k}\left(\widetilde{X}_{k}^{(W)}-\widetilde{X}_{k \mid k-1}^{(j, i)}\right), \\
\widetilde{K}_{k} & =I_{\mathrm{Num}} \Delta_{k \mid k-1}^{(j)}\left(I_{\mathrm{Num}} \widetilde{S}_{k}\right)^{-1}, \\
\widetilde{S}_{k} & =\Upsilon_{\mathrm{Num}}\left(H P_{k \mid k-1} H^{T}+\frac{R_{k}}{|W|}\right)+\Delta_{k \mid k-1}^{(j)},
\end{aligned}
$$

where $I_{\text {Num }}$ denotes the identity matrix with Num order. $R_{k}$ denotes the measurement noise of a measurement source. $\Upsilon_{\text {Num }}(P)$ denotes the decomposed function which can decompose the matrix $P$ as a set with Num variances. $\widetilde{X}_{k}^{(W)}=$ $\left[\left\{\widetilde{\rho}^{(W, i)}\right\}_{i=1}^{\text {Num }}\right]^{T}$ denotes the pseudocontrol point matrix which is used to update the control point matrix $\widetilde{X}_{k \mid k}^{(j, i)}$, where $\widetilde{\rho}^{(W, i)}$ can be obtained by

$$
\tilde{\rho}^{(W, i)}= \begin{cases}\frac{1}{\left|W^{(i)}\right|} \sum_{z \in W^{(i)}} \sqrt{\left(z-\bar{z}_{k}^{W}\right)^{T}\left(z-\bar{z}_{k}^{W}\right)}, & \left|W^{(i)}\right| \neq 0 \\ 0, & \left|W^{(i)}\right|=0,\end{cases}
$$

where $\left|W^{(i)}\right|$ denotes the measurement number of $i$ th angle direction in cell $W$.

Notice that the D-distance partition method [17] is implemented in the part of measurement partition. Set the maximum distance as the mean size of the shape, and it can be obtained by

$$
\tilde{\rho}_{k, \max }=\frac{\sum_{j=1}^{J_{k-1 \mid k-1}} w_{k-1 \mid k-1}^{(j)} \sum_{i=1}^{\mathrm{Num}} 2 \widetilde{\rho}_{k-1 \mid k-1}^{(j, i)}}{\operatorname{Num} \cdot \sum_{j=1}^{J_{k-1 \mid k-1}} w_{k-1 \mid k-1}^{(j)}} .
$$

Finally, shapes of multiple extended targets are extracted according to $X_{k+1}$ and the B-spline curve fitting technique described in Section 2.2.

\section{Simulations}

Assume that there is an extended target making a uniform motion in a two-dimensional simulation scenario, and the state equation and the measurement equation are the same as (1) and (2), respectively, where $x_{k}=\left[x, v_{x}, y, v_{y}\right]^{T}$ denotes the target state, which contains position and velocity information. The state transition matrix $F=\left[\begin{array}{llll}1 & 1 & 0 & 0 \\ 0 & 1 & 0 & 0 \\ 0 & 0 & 1 & 1 \\ 0 & 0 & 0 & 1\end{array}\right]$ and measurement matrix $H=\left[\begin{array}{ll}1 & 0 \\ 0 & 0 \\ 0 & 1 \\ 0 & 0\end{array}\right]^{T}$. The process noise covariance $Q_{k}=\operatorname{diag}\left[\sigma_{w 1}^{2}, \sigma_{w 2}^{2}\right]$ with $\sigma_{w 1}=\sigma_{w 2}=1$, and the measurement noise covariance $R_{k}=\operatorname{diag}\left[\sigma_{v 1}^{2}, \sigma_{v 2}^{2}\right]$ with $\sigma_{v 1}=\sigma_{v 2}=1$. The initial covariance $P_{0 \mid 0}=\operatorname{diag}[5,1,5,1]$ and the shape noise covariance is $\Delta_{0 \mid 0}=\underbrace{[1,1, \ldots, 1]^{T}}_{n}$. The other parameters $\aleph=\{(2 \pi / n) \cdot i\}_{i=0}^{n}, n=20, d=1.5$, and $m=45$. The way to generate measurements is the same as that of the RM method. The real measurement is assumed as the scattering center, that is, a measurement source. The observation measurements are generated from the scattering center with measurement noises. Notice that the measurement noise is assumed to be small compared to the target extent.

Example 1 (star-shaped extended target). Figure 2 shows the shape estimation by the proposed algorithm and the RM method [9], respectively. It is clear that the proposed algorithm has a higher accuracy than that of the RM method. 

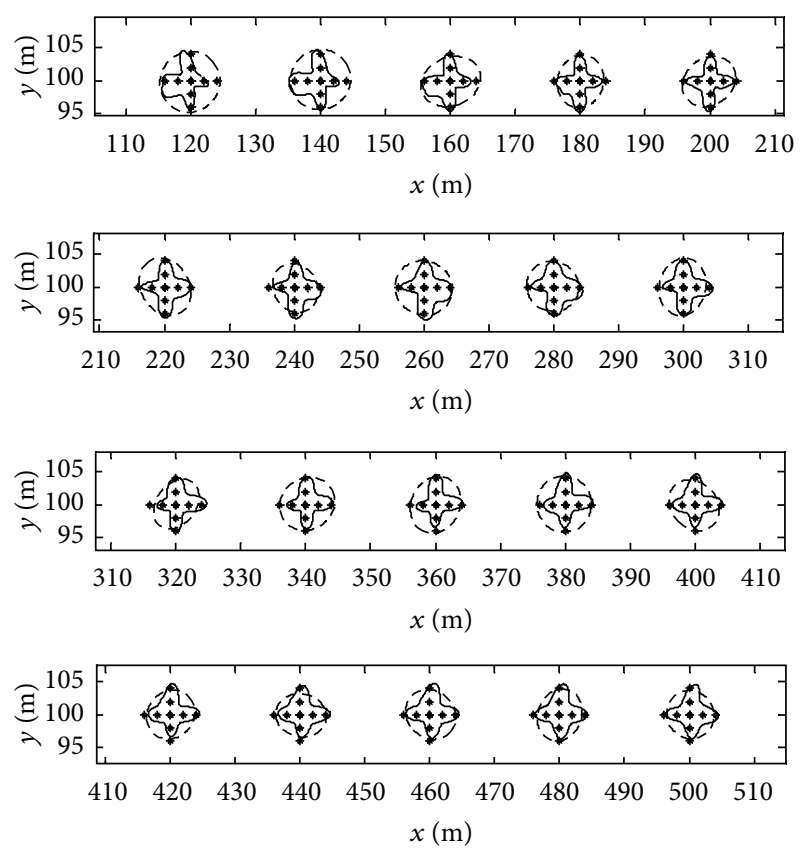

* Real measurement

— Proposed algorithm

- - RM method

FIGURE 2: Shape estimation by the proposed algorithm and the RM method.

The reason is that the RM method can only estimate the elliptical shape of the target.

Figure 3 shows the measurements of the extended target from the 1st frame to the 20th frame. As can be seen, the measurement noise is big which makes the shape hard to be estimated from the single frame. However, the proposed algorithm can extract the accurate shape by multiple frame statistic technique and the B-spline curve fitting. It is shown that the proposed algorithm has a good capacity of resisting disturbance of the noise.

Figure 4 shows the average shape estimate. It is clear that the proposed algorithm can obtain the shape features effectively.

Example 2 (Y-shaped extended target). Figure 5 shows the shape estimation by the proposed algorithm and the RM method, respectively. Figure 6 shows the measurements of the extended target from the 1st to the 20th frames. Figure 7 shows the average shape estimate. As can be seen, the proposed algorithm has a better performance for shape estimate of irregular extended targets.

Example 3 (multiple extended target tracking). The scenario of multiple extended target tracking is the same as that of [17]. There are four targets and two of them cross at time $k=56$, and one target is spawned at time $k=$ 66. Assume that the measurement noise covariance and the process noise covariance are $R_{k}=\operatorname{diag}[0.7,0.7]$ and $Q_{k}=\operatorname{diag}[1,1]$, respectively. Shape noise covariance is
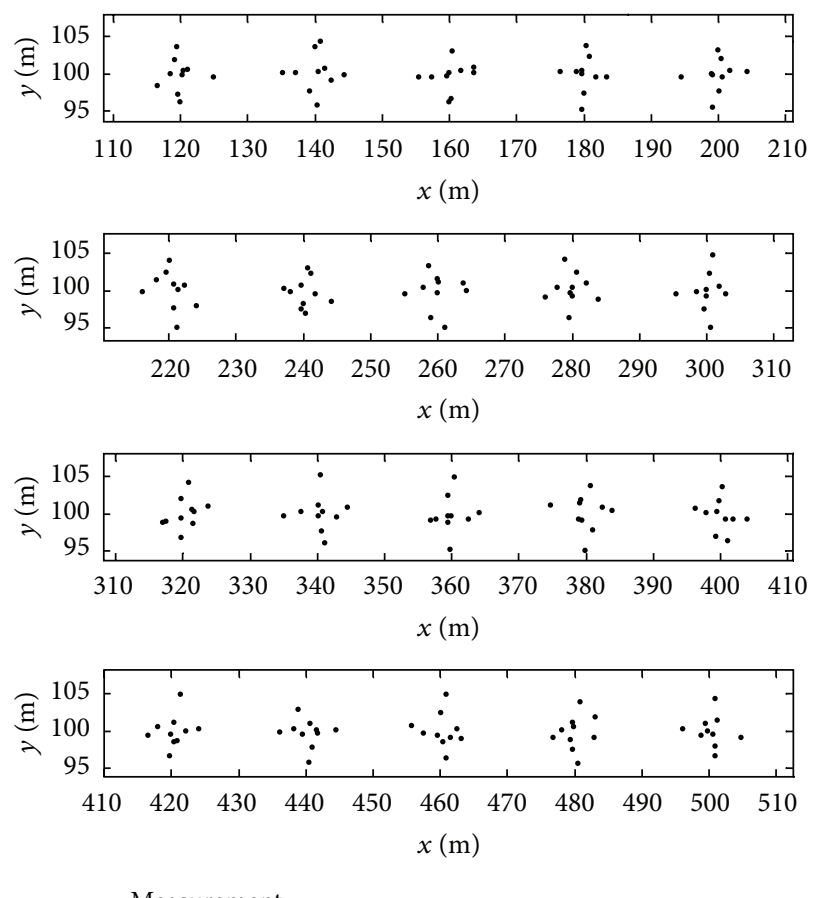

Figure 3: The real measurements of the extended target (1 20 frames).

$\mathbb{Q}_{k}=\underbrace{[0.05,0.05, \ldots, 0.05]}_{\text {Num }}, \mathrm{Num}=12$, and $\omega=0.01$. The probabilities of target survival and detection are $p_{S}=0.99$ and $p_{D}=0.99$, respectively. The birth process is modeled on a Poisson RFS with Gaussian mixture intensity:

$$
\begin{aligned}
D_{b}(x, X)= & \sum_{j=1}^{2} \omega_{b}^{(j)} N\left(x \mid m_{b}^{(j)}, P_{b}^{(j)}+\bar{\sigma}_{b} \widetilde{I}\right) \\
& \times \prod_{i=1}^{N u m} N\left(X_{b}^{(j)} \mid \widetilde{X}_{b}^{(j, i)}, \Delta_{b}^{(j, i)}\right),
\end{aligned}
$$

where $m_{b}^{(1)}=[250 \mathrm{~m}, 250 \mathrm{~m}, 0 \mathrm{~m} / \mathrm{s}, 0 \mathrm{~m} / \mathrm{s}]^{T}, m_{b}^{(2)}=[-250 \mathrm{~m}$, $-250 \mathrm{~m}, 0 \mathrm{~m} / \mathrm{s}, 0 \mathrm{~m} / \mathrm{s}]^{T}, P_{b}^{(1)}=P_{b}^{(2)}=\operatorname{diag}[100,100,15,15]$, and $\omega_{b}^{(1)}=\omega_{b}^{(2)}=0.1, \widetilde{X}_{b}^{(1,1)}=\cdots=\widetilde{X}_{b}^{(1, \text { Num })}=\widetilde{X}_{b}^{(2,1)}=\cdots=$ $\widetilde{X}_{b}^{(2, \text { Num })}=5, \Delta_{b}^{(1,1)}=\cdots=\Delta_{b}^{(1, \text { Num })}=\Delta_{b}^{(2,1)}=\cdots=$ $\Delta_{b}^{(2, \text { Num })}=1$.

The intensity of the spawned targets is $v_{\beta}(x)=$ $0.05 N\left(x \mid \xi, Q_{\beta}\right) \times \prod_{i=1}^{N u m} N\left(X \mid \xi, \Delta_{\beta}\right)$, where $Q_{\beta}=$ $\operatorname{diag}[100,100,400,400]$ and $\Delta_{\beta}=\underbrace{[1,1, \ldots, 1]}_{\text {Num }}$.

Figure 8 (a) shows the tracking results of the targets in the whole tracking area, and Figures $8(\mathrm{~b}), 8(\mathrm{c})$, and 8(d) show the shape estimates of different targets in special tracking area. As can be seen from Figure 8(b), the shapes of Targets 1 and 2 are assumed as ellipses; although they are not accurate, they can be updated approximately to the real shapes of the targets at several time points. The reason is that the shape parameters in the proposed shape estimate method are updated at each time 


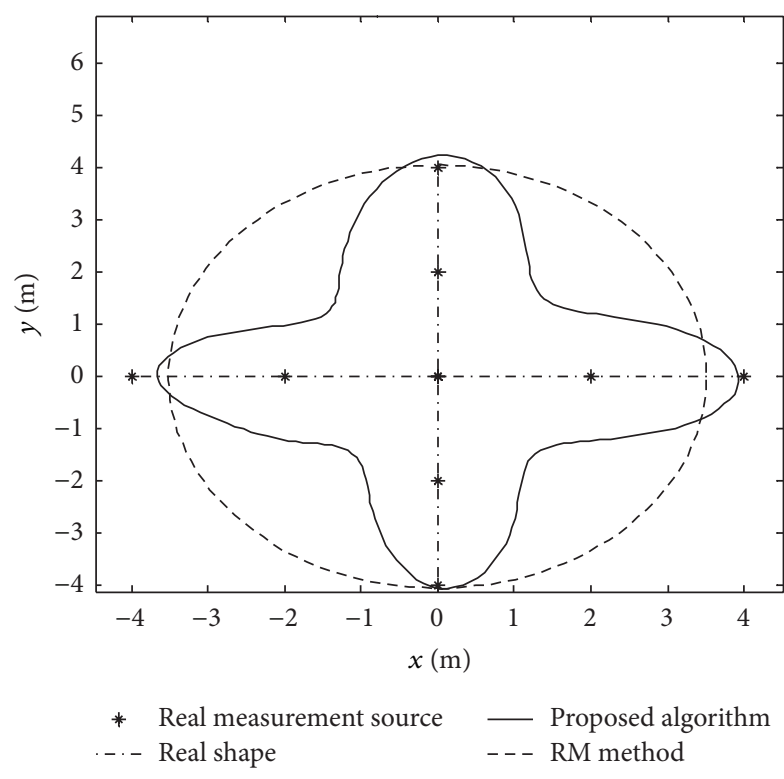

FIgURE 4: Average shape estimate.
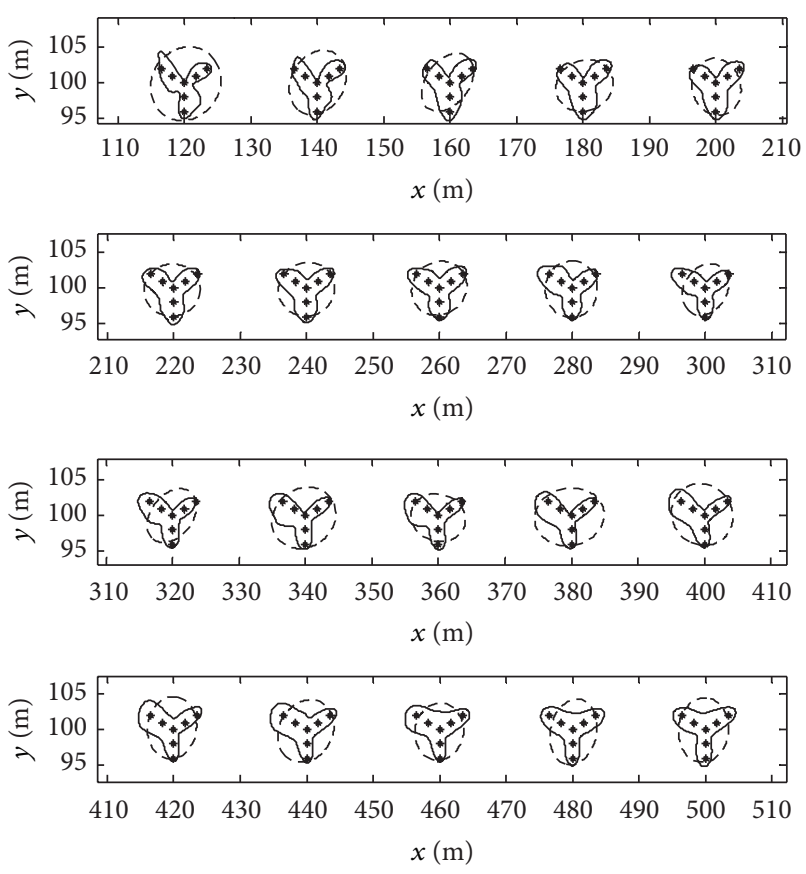

* Real measurement

— Proposed algorithm

--- RM method

FIGURE 5: Shape estimation by the proposed algorithm and the RM method.

when the latest measurements arrive. Moreover, the Gaussian mixture technique is employed, which can approximately fit the real shape distribution of the extended target. In Figure $8(\mathrm{c})$, we can see that the shape estimates are not accurate when the targets cross each other, but they can also be updated approximately to the real target shapes. Figure $8(\mathrm{~d})$ shows the shape estimate of a spawned target;
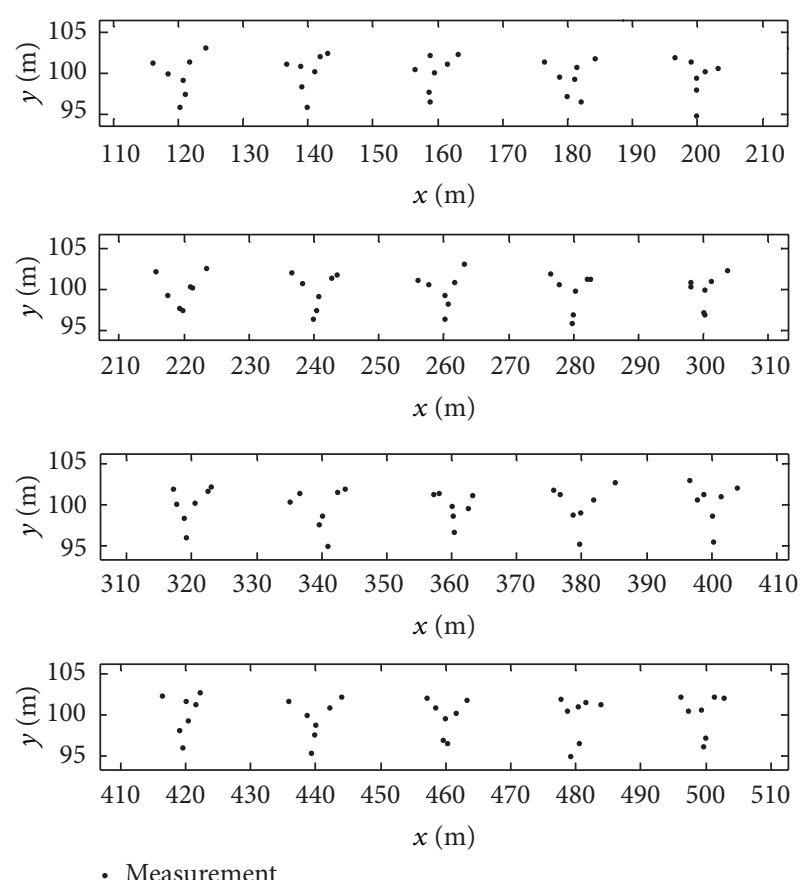

FIgURE 6: The real measurements of the extended target (1 20 frames).

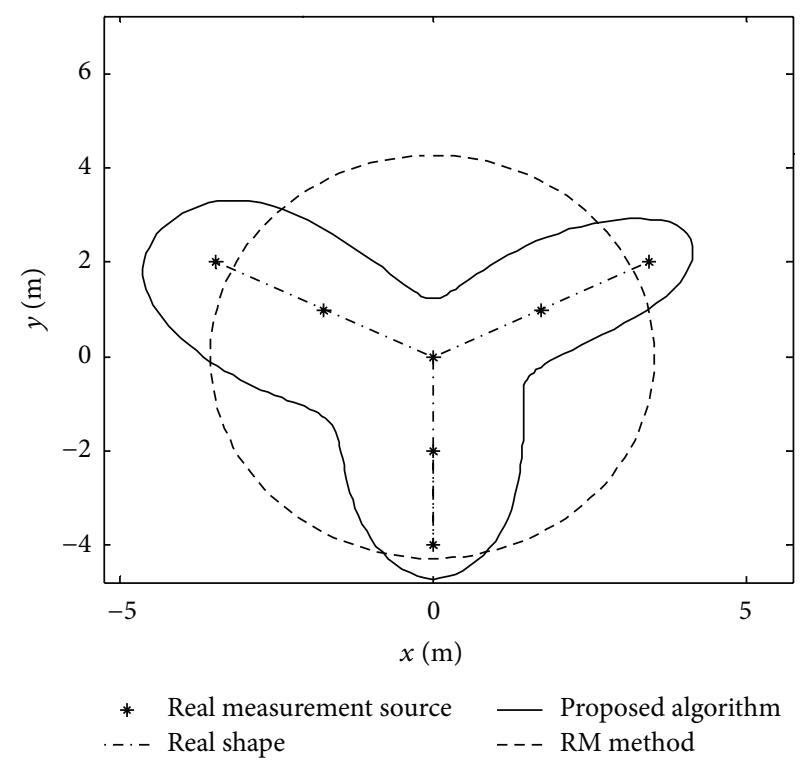

Figure 7: Average shape estimate.

it is showed that the proposed algorithm also has a good performance for shape estimate of the spawned target.

Figure 9 shows the number estimates and Figure 10 shows the accuracy statistic by the OSPA distance [18]. Notice that OSPA distance sharply increases at 56th and 66th seconds. The reason is that the targets make a cross with each other at 56th second and a spawned target appears. Generally, we can see that the proposed algorithm has a good performance for multiple extended target tracking with shape estimates. 


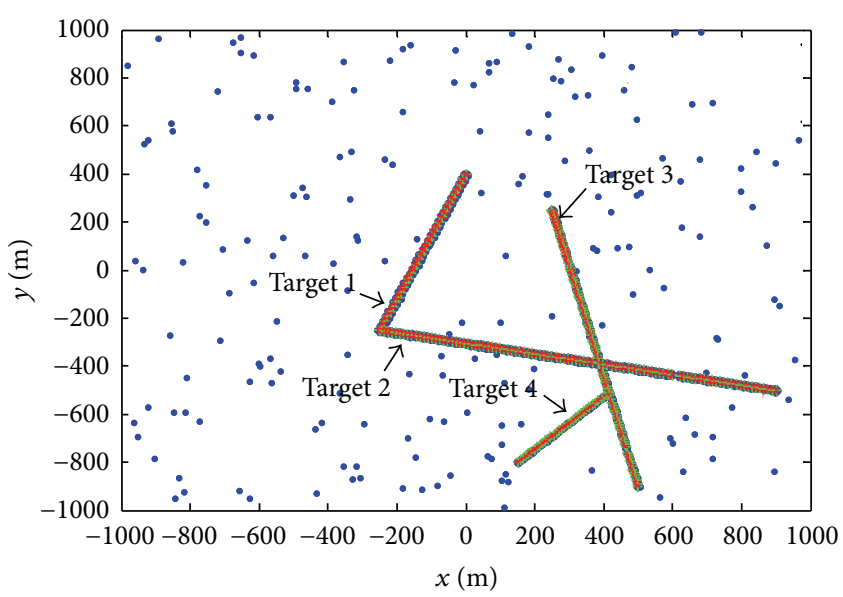

(a) Measurements and tracking results

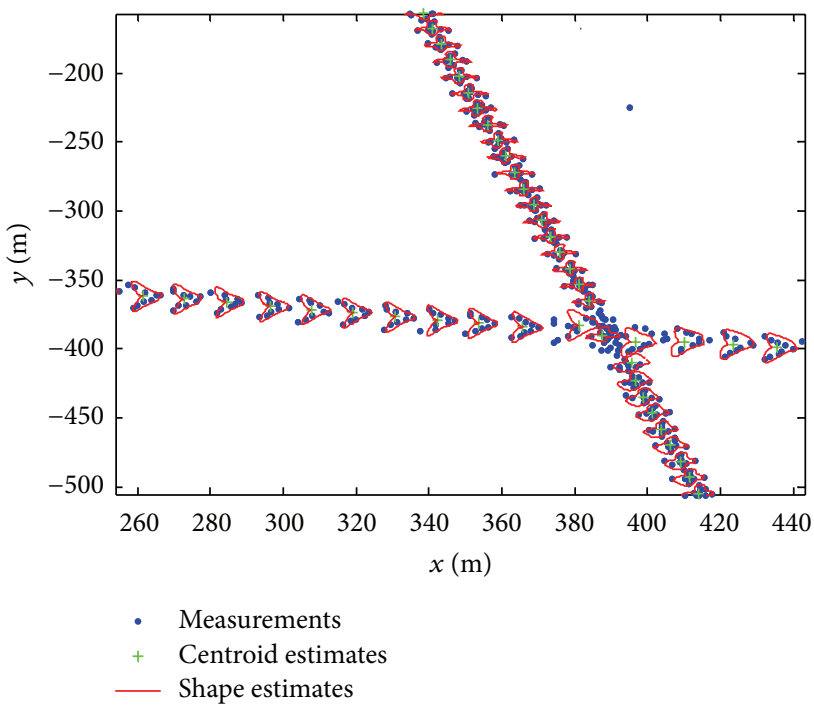

(c) Shape estimates of cross targets

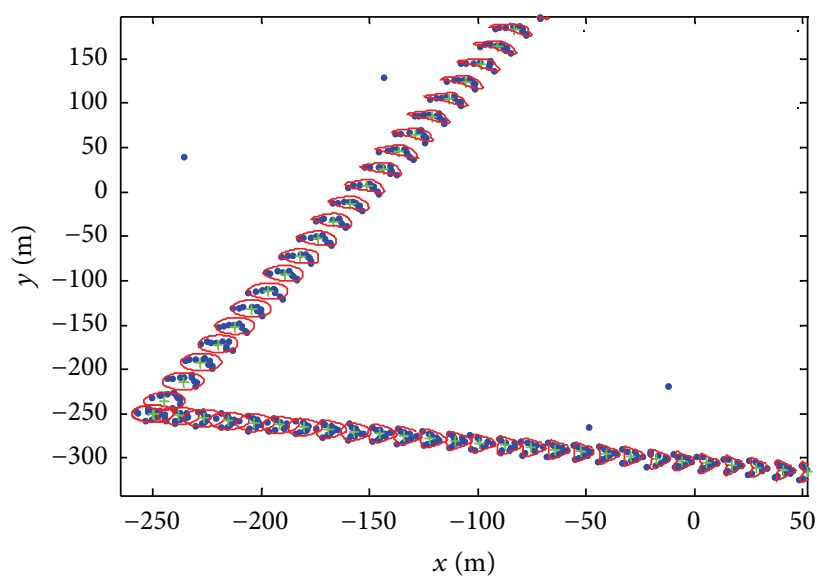

(b) Shape estimates of Targets 1 and 2

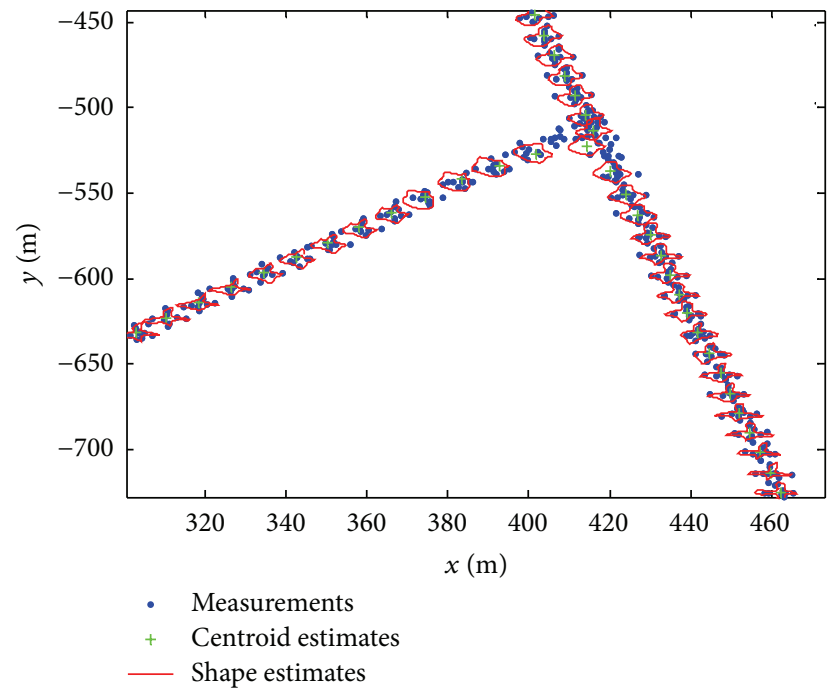

(d) Shape estimates of the spawned target

FIGURE 8: Shape estimate results.

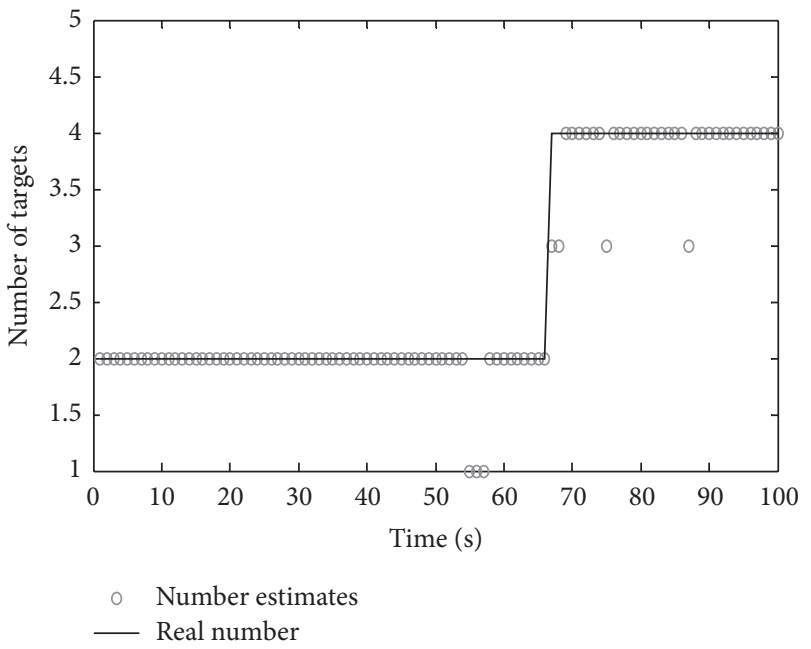

FIGURE 9: Number estimates.

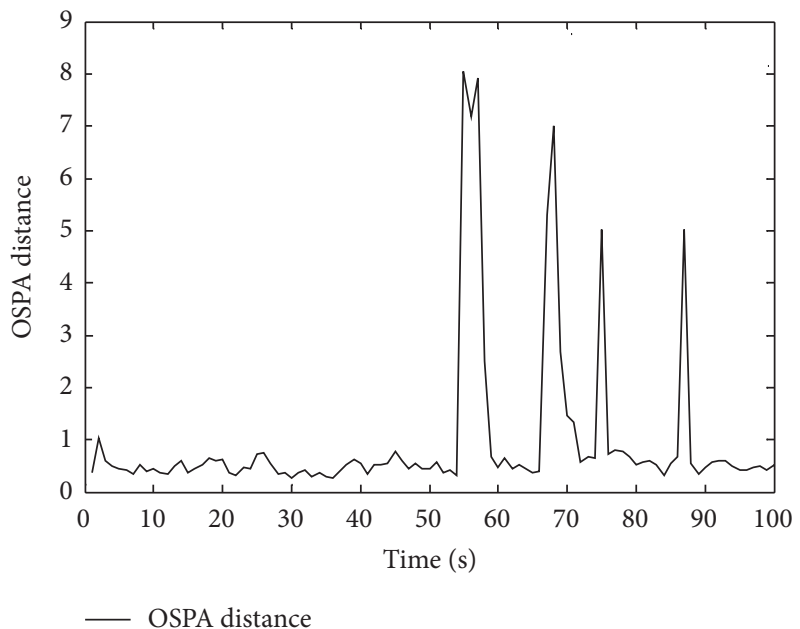

Figure 10: OSPA distance. 


\section{Conclusions}

In this paper, a novel shape estimation algorithm is proposed based on the B-spline curve fitting. The multiple frame statistic technique is introduced to construct the pseudomeasurement sets. The selected control points are used to form the B-spline curve, and then the curve yields the shapes of the extended targets. Moreover, the proposed shape estimation algorithm is modified suitably and combined with the probability hypothesis density (PHD) filter for multiple extended target tracking. Simulations show that the proposed algorithm has a good performance for shape estimates of extended targets.

\section{Conflict of Interests}

The authors declare that there is no conflict of interests regarding the publication of this paper.

\section{Acknowledgments}

This paper is supported by the National Natural Science Foundation of China (nos. 61305017 and 61304264) and the Natural Science Foundation of Jiangsu Province (no. BK20130154).

\section{References}

[1] K. Gilholm, S. Godsill, S. Maskell, and D. Salmond, "Poisson models for extended target and group tracking," in Signal and Data Processing of Small Targets, Proceedings of the SPIE, pp. 1-12, San Diego, Calif, USA, August 2005.

[2] K. Gilholm and D. Salmond, "Spatial distribution model for tracking extended objects," IEE Proceedings Radar, Sonar and Navigation, vol. 152, no. 5, pp. 364-371, 2005.

[3] M. Baum and U. D. Hanebeck, "Random hypersurface models for extended object tracking," in Proceedings of the 9th IEEE International Symposium on Signal Processing and Information Technology (ISSPIT '09), pp. 178-183, Ajman, United Arab Emirates, December 2009.

[4] M. Baum and U. D. Hanebeck, "Shape tracking of extended objects and group targets with star-convex RHMs," in Proceedings of the 14th International Conference on Information Fusion (Fusion '11), pp. 1-8, Chicago Ill, USA, July 2011.

[5] R. Mahler, "PHD filters for nonstandard targets, I: extended targets," in Proceedings of the 12th International Conference on Information Fusion (FUSION '09), pp. 915-921, Seattle, Wash, USA, July 2009.

[6] K. Granstrom, C. Lundquist, and O. Orguner, "Extended target tracking using a gaussian-mixture PHD filter," IEEE Transactions on Aerospace and Electronic Systems, vol. 48, no. 4, pp. 3268-3286, 2012.

[7] K. Granstrom and U. Orguner, "A PHD filter for tracking multiple extended targets using random matrices," IEEE Transactions on Signal Processing, vol. 60, no. 11, pp. 5657-5671, 2012.

[8] J. W. Koch, "Bayesian approach to extended object and cluster tracking using random matrices," IEEE Transactions on Aerospace and Electronic Systems, vol. 44, no. 3, pp. 1042-1059, 2008.
[9] M. Feldmann, D. Fränken, and W. Koch, "Tracking of extended objects and group targets using random matrices," IEEE Transactions on Signal Processing, vol. 59, no. 4, pp. 1409-1420, 2011.

[10] B. Ristic, S. Arulampalm, and N. J. Gordon, Beyond the Kalman Filter: Particle Filters for Tracking Applications, Artech House, 2004.

[11] H. Haron, A. Rehman, D. I. S. Adi, S. P. Lim, and T. Saba, "Parameterization method on B-spline curve," Mathematical Problems in Engineering, vol. 2012, Article ID 640472, 22 pages, 2012.

[12] H. Park, "B-spline surface fitting based on adaptive knot placement using dominant columns," Computer Aided Design, vol. 43, no. 3, pp. 258-264, 2011.

[13] B. Vo and W. Ma, “The Gaussian mixture probability hypothesis density filter," IEEE Transactions on Signal Processing, vol. 54, no. 11, pp. 4091-4104, 2006.

[14] W. L. Li and Y. M. Jia, "Gaussian mixture PHD filter for jump Markov models based on best-fitting Gaussian approximation," Signal Processing, vol. 91, no. 4, pp. 1036-1042, 2011.

[15] J. J. Yin, J. Q. Zhang, and Z. S. Zhuang, "Gaussian sum PHD filtering algorithm for nonlinear non-Gaussian models," Chinese Journal of Aeronautics, vol. 21, no. 4, pp. 341-351, 2008.

[16] K. Granström and U. Orguner, "A PHD filter for tracking multiple extended targets using random matrices," IEEE Transactions on Signal Processing, vol. 60, no. 11, pp. 5657-5671, 2012.

[17] K. Granström, C. Lundquist, and U. Orguner, "A Gaussian mixture PHD filter for extended target tracking," in Proceedings of the 13th Conference on Information Fusion, pp. 1-8, Edinburgh, Scotland, July 2010.

[18] D. Schuhmacher, B. T. Vo, and B. N. Vo, "A consistent metric for performance evaluation of multi-object filters," IEEE Transactions on Signal Processing, vol. 56, no. 8, part 1, pp. 3447-3457, 2008. 


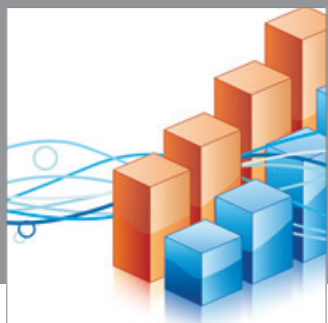

Advances in

Operations Research

mansans

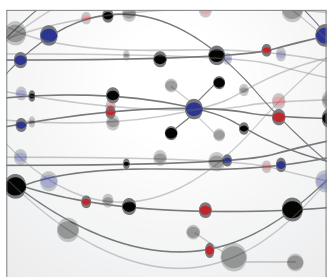

The Scientific World Journal
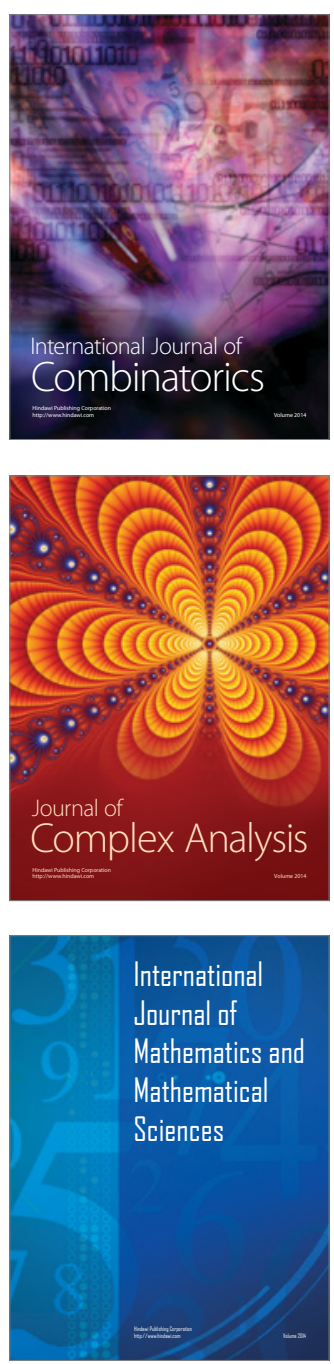
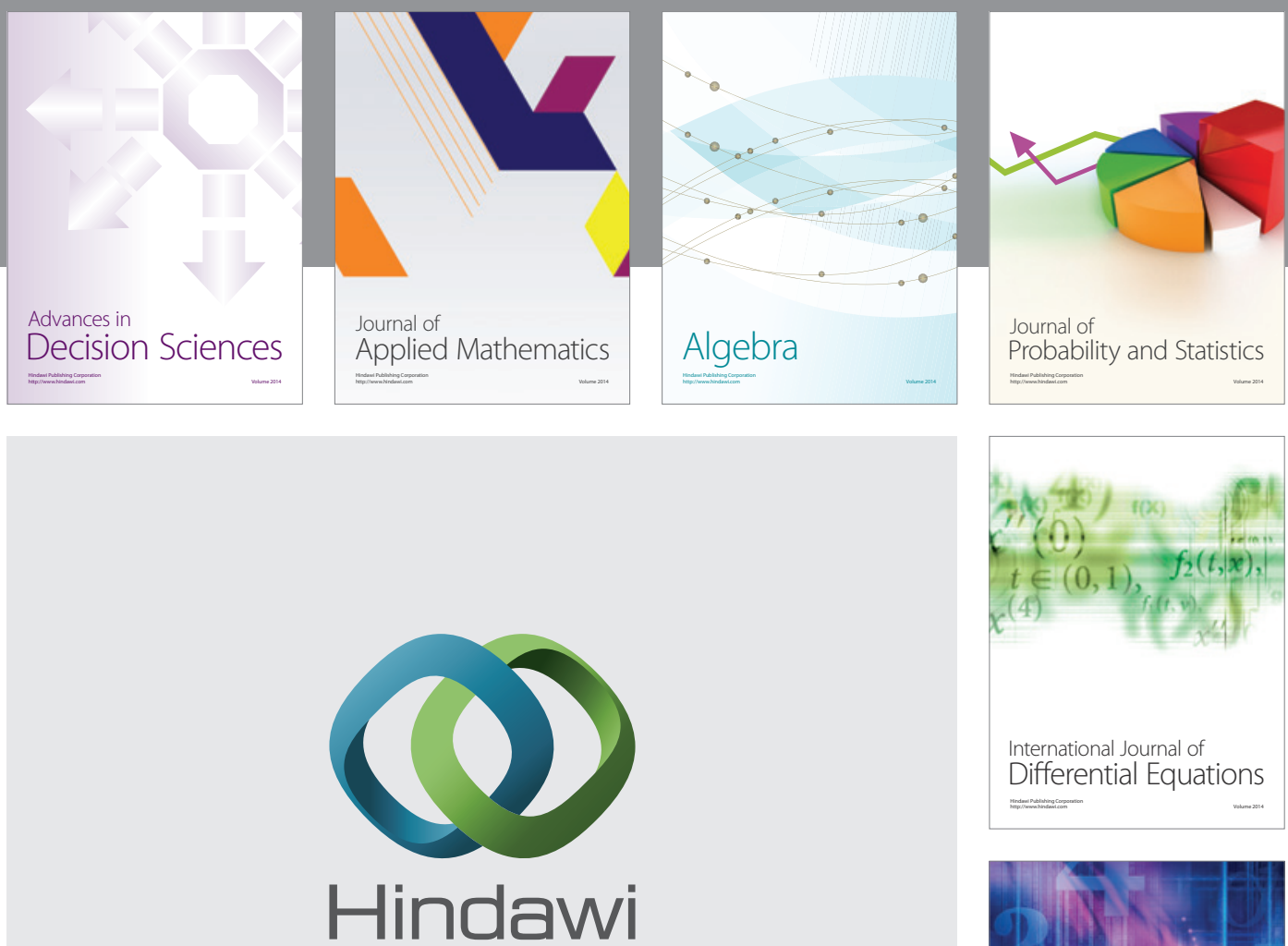

Submit your manuscripts at http://www.hindawi.com
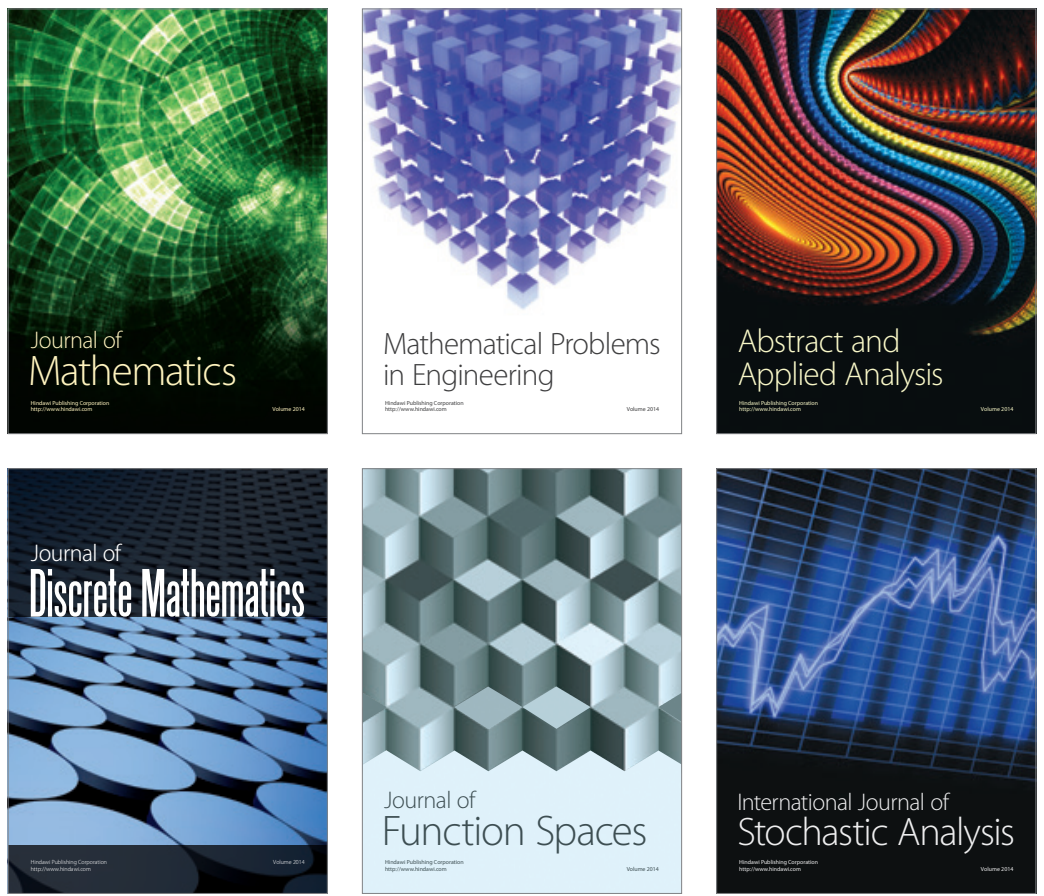

Journal of

Function Spaces

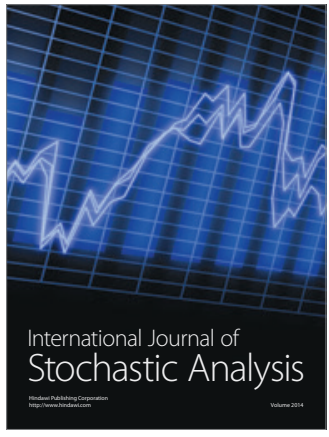

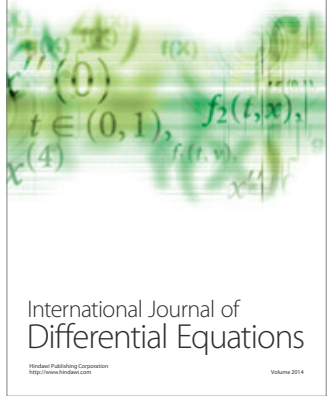
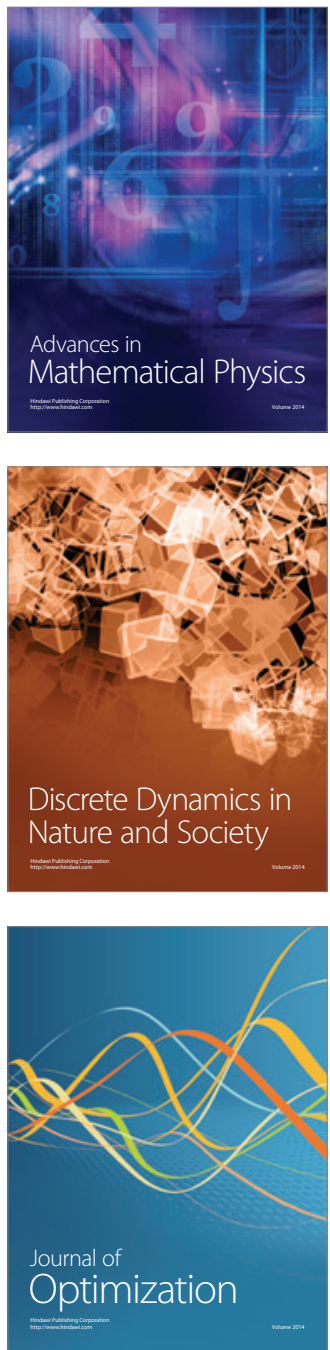